\title{
Neurobiological Modeling of Bursting Response During Visual Attention
}

\author{
Reza Rajimehr, Leila Montaser Kouhsari \\ Institute for Studies in Theoretical Physics and Mathematics, Tehran
}

\begin{abstract}
Thalamic neurons have an exclusive property named bursting response. Bursting response seems to have a critical role in producing saliency map and encoding conspicuity of locations during visual attention. Attention window is developed in thalamus due to its retinotopic organization. The global competitive network in thalamic reticular nucleus (NRT) determines which thalamic cells should produce bursting response. These cells are corresponded with attention window or attended location in the visual field. The computational procedure of bursting response is studied by the means of neurobiological modeling of thalamic neurons and their bimodal behavior (periodic bursting and periodic spiking patterns). The effect of NRT on thalamic relay neurons is considered in each mode of thalamic response. We also achieved the results of modeling through computer simulation of bimodal behavior in thalamic neurons.
\end{abstract}

Keywords: Thalamic relay neuron, Bursting response, Visual attention, Model

\section{Introduction}

As Jahnsen and Llinás $(1,2,3)$ showed, we understand that all thalamic relay neurons (TRN) have two distinct modes of behavior. First when the cell is near its normal resting potential $(-60 \mathrm{mv})$, second when it is hyperpolarized and its potential is near - $70 \mathrm{mv}$. The former responds to an injected current by firing at a rate between 25 and 100 spikes per second, which increases with greater values of the injected current, and later responds to an injected current, after a short delay, with fast burst of spikes and firing rates nearer 300 spikes per second. Because of aftereffect, the cell will not produce another bursting response although the injected current is constant. $\mathrm{Ca}^{2+}$ - dependent $\mathrm{K}^{+}$channels are responsible for bursting behavior. Other channels related to this phenomenon are low threshold and high threshold $\mathrm{Ca}^{2+}$ currents, early $\mathrm{K}^{+}$current and $\mathrm{Na}^{+}$current. The rhythmic behavior of these ion currents is described as follows.

When the cell membrane is hyperpolarized for at least $150 \mathrm{msec}$, a low threshold $\mathrm{Ca}^{2+}$ current is de-inactivated, which normally inactivated at the membrane resting potential (or at potentials more positive than the membrane resting potential). This current is probably localized at the level of the soma. The low threshold spike leads to the activation of a high threshold $\mathrm{Ca}^{2+}$ current (probably localized at the dendritic level). Influx of $\mathrm{Ca}^{2+}$ increases the excitatory state of the relay neuron. In this state, 
the neuron produces action potentials due to $\mathrm{Na}^{+}$current. Each action potential is followed by a phase of after-hyperpolarization in result of $\mathrm{Ca}^{2+}$ - dependent $\mathrm{K}^{+}$ currents. This after-hyperpolarization is weak so the neuron can fire bursts of action potentials at higher frequencies. The early $\mathrm{K}^{+}$current prolongs the duration of the hyperpolarization. The responses of thalamic neurons to excitatory afferents depend on the value of the resting membrane potential, i.e. on the activity of the inhibitory afferent pathways. This periodic pattern will continue provided that the input current to the neuron is available. The periodic bursting pattern based on ion currents is studied and simulated in this article.

Taylor and Alavi suggested NRT as a Winner Take All (WTA) network during visual attention. NRT has a global competitive network in which long-range inhibition occurs so corresponding neurons with attention window can inhibit other neurons (4). These neurons send inhibitory projections to the thalamus.

Crick believes that inhibition of NRT on corresponding thalamic neurons in attention window enables these neurons to produce bursting response. In Crick's theory (5), the retinotopic area for attention window is thalamus in which a number of thalamocortical pathways are activated more than other pathways during spot light attention. As a result, transmission of information is facilitated through these activated thalamocortical projections. Thalamus and NRT coupled nets have a role in producing saliency map and implementing the WTA network.

\section{Model}

RT GABAergic neurons hyperpolarize TR neurons, which inhibitory post-synaptic potential (IPSP) is made by $\mathrm{K}^{+}$current $\left(\mathrm{K}^{+}\right.$dependant IPSP is mediated by GABA B receptors). Hyperpolarization increases the threshold of TR neuron.

Let $?_{i}$ denote the threshold of TR neuron, which is altered by two independent factors:

$$
\theta_{i_{\text {total }}}(t)=\theta_{i}^{1}(t)+\theta_{i}^{2}(t)
$$

Where $\boldsymbol{\theta}_{\boldsymbol{i}}^{1}(\boldsymbol{t})$ shows the alternation of the threshold by NRT inhibition and $\boldsymbol{\theta}_{\boldsymbol{i}}^{2}(\boldsymbol{t})$ shows the effect of neuronal habituation on the threshold. The initial total threshold of TR neuron is $m\left(\boldsymbol{\theta}_{i}^{1}(0)=0\right.$ and $\left.\boldsymbol{\theta}_{i}^{2}(0)=\boldsymbol{m}\right)$.

$\boldsymbol{\theta}_{i}^{1}(\boldsymbol{t})$ is calculated by stationary solution to below differential equation:

$$
\varepsilon \frac{d \theta_{i}^{1}}{d t}=-\theta_{i}^{1}+\left(G_{k}(t)-G_{k}(0)\right)
$$


Where $G_{k}(t)$ is the amount of $\mathrm{K}^{+}$outward current or cellular channel permeability to $\mathrm{K}^{+}$, which decreases the amount of intra-cellular $\mathrm{K}^{+}$concentration. Increasing of $G_{k}(t)$ enhances the neuronal threshold. $G_{k}(t)$ is itself altered by below differential equation:

$$
\varepsilon \frac{d G_{k}}{d t}=-G_{k}+\psi(t) \cdot \phi \cdot Z_{i}
$$

Where $F . Z_{i}$ is the inhibition of NRT on TR neuron. ?(t) is a periodic square function, which alters between 0 and 1 . At the beginning $(t=0)$, NRT sends signals to thalmus and ?( 0$)$ equals 1 so $G_{k}(t)$ increases. As we will discuss later, transient hyperpolarization of the membrane (by high amo unt of $G_{k}(t)$ ) for at least $150 \mathrm{msec}$ increases the cellular channel permeability to $\mathrm{Ca}^{2+}\left(G_{c a}\right)$ and de-inactivates a low threshold transient $\mathrm{Ca}^{2+}$ current. The low threshold spike leads to the activation of a high threshold $\mathrm{Ca}^{2+}$ current in turn. Increase in the intra-cellular $\mathrm{Ca}^{2+}$ concentration enhances the saturation point of TR neuron's activity $(A)$ as an index of maximal firing rate (the amount of $A$ directly depends on $G_{k}(t)$ with considering phase locking between them). When $A$ reaches a value named $A_{\max }$, then ? (t) becomes zero so $G_{k}(t)$ gradually decreases until $A$ attracts to the point of $A_{\min }$. In this phase, hyperpolarization is removed and $\mathrm{K}^{+}$ions enter to the neuron by the help of electrogenic pumps. At the point of $A_{\min }$, ? $(t)$ equals 1 , which causes $G_{k}(t)$ to increase. ? $(t)$ is not changed until $A$ becomes $A_{\text {max }} . \mathrm{Ca}^{2+}$-dependent $\mathrm{K}^{+}$currents are activated at the time of $A_{\max }$ because of phase locking between $A$ and $G_{k}(t)$. The early $\mathrm{K}^{+}$current also prolongs the duration of hyperpolarization. The procedure of described oscillation continues if NRT sends inhibitory signals continuously. Oscillatory behavior of the bursting phenomenon is due to after-hyperpolarization, which follows action potentials at the point of $A_{\max }$.

Hyperpolarization of the TR neuron due to $\mathrm{K}^{+}$current can lead to $\mathrm{Ca}^{2+}$ current activation in this neuron. The development of cellular channel permeability to $\mathrm{Ca}^{2+}$ can be shown as:

$$
\varepsilon \frac{d G_{c a}}{d t}=-G_{c a}+\left(\theta_{i}^{1}(t)-\theta_{i}^{1}(0)\right)
$$

Influx of $\mathrm{Ca}^{2+}$ into TR neuron increases its excitatory state. In other words, the saturation point of neuronal activity is enhanced by below differential equation:

$$
\varepsilon \frac{d A}{d t}=-A+\eta \cdot\left(G_{c a}(t)-G_{c a}(0)\right)
$$

Where? is a positive constant value indicating the amount of increase in $A$. If ? has a small value, the alternation of $A$ will be less than $\boldsymbol{\theta}_{i}^{1}(\boldsymbol{t})$ so the bursting response is produced only in the presence of remarkable NRT inhibition (see Eq.(12)). 
Oscillatory diagrams of $G_{k}(t), \boldsymbol{\theta}_{\boldsymbol{i}}^{1}(\boldsymbol{t}), G_{c a}(t)$ and $A(t)$ in TR neuron during NRT inhibition are shown in Fig. (1.a).

Let $g\left(Y_{i}\right)$ denote the output of TR neuron as a sigmoidal function, which is increased by $A$ (for $Y_{i}=\boldsymbol{\theta}_{\boldsymbol{i}_{\text {total }}}(\boldsymbol{t})$ ). $A$ is the saturation point of the sigmoidal function of TR neuron's output and $g\left(Y_{i}\right)$ is the amount of firing rate. At the $A_{\max }$, the firing rate has a great value near bursting frequency, which becomes maximal at the $A_{u p}\left(A_{u p}\right.$ is the upper limit of the oscillation, which is made after $A_{\max }$ due to the dynamical property of the system). Then the amount of $A$ and firing rate decreases. This is the same as aftereffect of bursting response or gradually reset of TR neurons and their specific conductances because of the after-hyperpolarization effect (1). The apparition time of after-hyperpolarization (in result of early $\mathrm{K}^{+}$current) depends on $A$. For $\boldsymbol{A}>\boldsymbol{A}_{\mathbf{m a x}}$, this aftereffect begins after a delay in increasing phase of $G_{k}(t)$ and decreasing phase of $A$, which is calculated as:

$\Delta t=t_{\left(A_{u p}\right)}-t_{\left(A_{\max }\right)}$

In this period, TR neuron produces some action potentials (in result of $\mathrm{Na}^{+}$current) with variable firing rate. $g\left(Y_{i}\right)$ will equal zero from $\boldsymbol{t}_{\boldsymbol{A}_{\mathbf{m a x}}}$ in decreasing phase of $A$ in its oscillation to $\boldsymbol{t}_{\boldsymbol{A}_{\text {max }}}$ in increasing phase of $A$ in later period, hence TR neuron will not produce the response in this time interval and the firing occurs after a delay when $A$ gets close to the $A_{\max }$. This response pattern of TR neuron is named Hopf/Hopf bursting (6) because $A$ increases the firing rate from $A_{\text {max }}$ to $A_{u p}$. The periodic bursting pattern is shown in Fig. (2.a).

If the inhibition of NRT $Z_{i}$ ) is low, bursting response pattern is not produced because $Z_{i}$ can not shift $A$ from its lower limit to upper limit. In this case, $G_{k}(t)$, $\boldsymbol{\theta}_{\boldsymbol{i}}^{1}(\boldsymbol{t}), G_{c a}(t)$ and $A(t)$ have a limit point attractor (see Fig. (1.b)) so $g\left(Y_{i}\right)$ will be constant and TR neuron will produce periodic spiking pattern with low firing rate provided that $Y_{i}=\boldsymbol{\theta}_{\boldsymbol{i}_{\text {total }}}(\boldsymbol{t})$ (see Fig. (2.b)). For $\boldsymbol{A}<\boldsymbol{A}_{\mathbf{m a x}}, ? t$ will be zero and refractory period exists after each action potential. In the TR neuron as a dynamical system, $Z_{i}$ acts as a bifurcation parameter by which the neuronal response pattern is fluctuated between two stable states (cycle and point basins of attraction).

The amount of critical $Z_{i}$, which the state of neuronal response is fluctuated around it, could be achieved by a dynamical analysis. Consider that $G_{k}$ directly influences $A$ (provided that the time constant values of $\boldsymbol{\theta}_{\boldsymbol{i}}^{1}(\boldsymbol{t})$ and $G_{c a}(t)$ functions are small) so Eq.(7) is obtained by substituting Eq.(3) in Eq.(5):

$$
A=A_{0}+\eta \cdot\left(G_{k}(t)-G_{k}(0)\right) \cdot\left(1-e^{-t / \varepsilon}\right)
$$

$A$ reaches $A_{\max }$ when:

$$
G_{k}(t)=G_{k}^{A_{m x}}=\frac{A_{\max }-A_{0}}{\eta \cdot\left(1-e^{-t / \varepsilon}\right)}+G_{k}(0)
$$




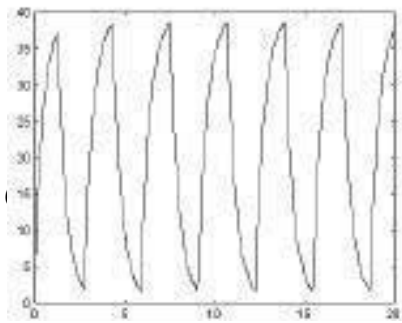

Time

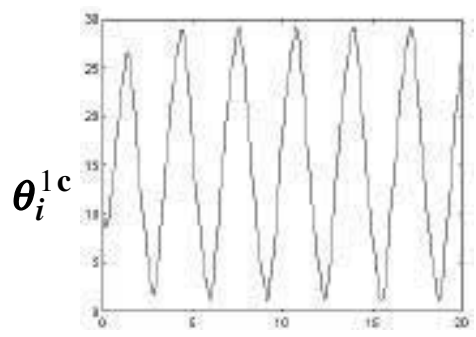

Time

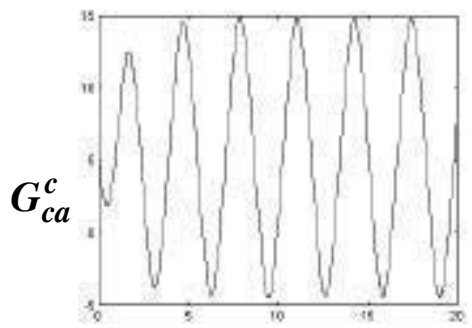

Time

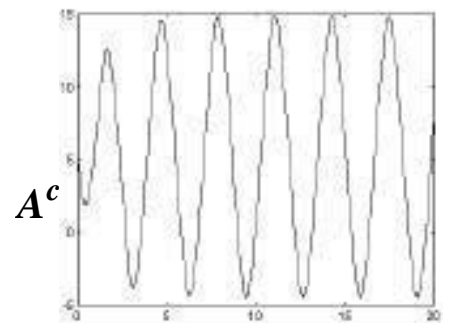

Time

(a)

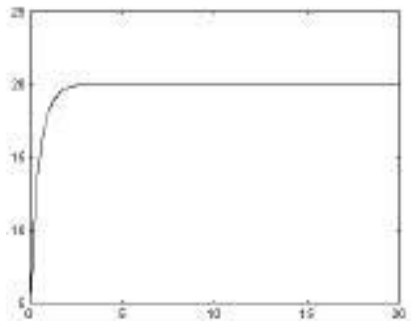

Time

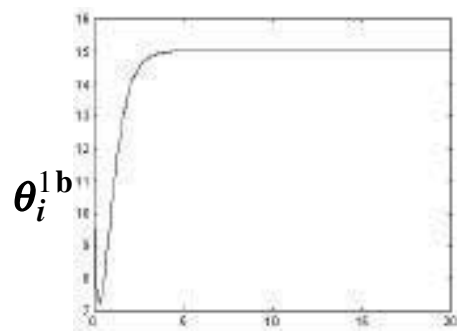

Time

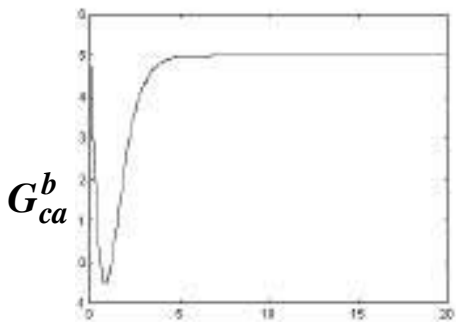

Time

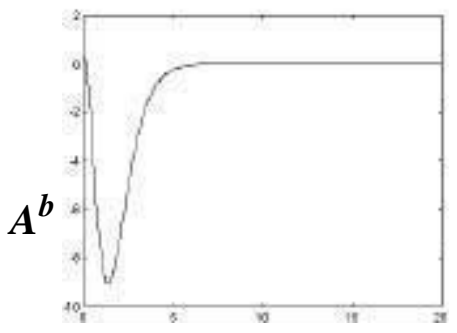

Time

(b)

Fig. 1. The results of computer simulation for values of $G_{k}(t), \boldsymbol{\theta}_{\boldsymbol{i}}^{1}(\boldsymbol{t}), G_{c a}(t)$ and $A(t)$ in different basins of attraction (cycle (a) and point (b)). 
Then $G_{k}(t)$ decreases exponentially $(?(t)=0)$ until $G_{k}(t)=G_{k}(0)$. In this point, the sign of derivative of $A$ is changed so that upper limit of $A$ in its oscillation is produced. Upper limit point of $A$ is computed as follows:

$$
A_{u p}=A_{\max }+\eta \cdot \operatorname{Max}\left[G_{k}^{A_{m x}} \cdot e^{-t / \varepsilon}-G_{k}(0)\right] \cdot\left(1-e^{-t / \varepsilon}\right)
$$

Then $A$ begins to decrease until reaches $A_{\min }$. At this point, $G_{k}(t)$ will be:

$$
G_{k}(t)=G_{k}^{A_{m n}}=\frac{A_{\min }-A_{u p}}{\eta \cdot\left(1-e^{-t / \varepsilon}\right)}+G_{k}(0)
$$

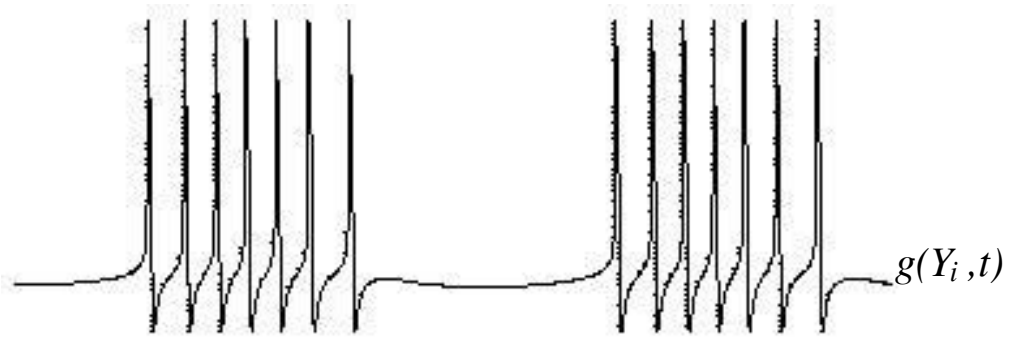

\section{Periodic Bursting}

(a)

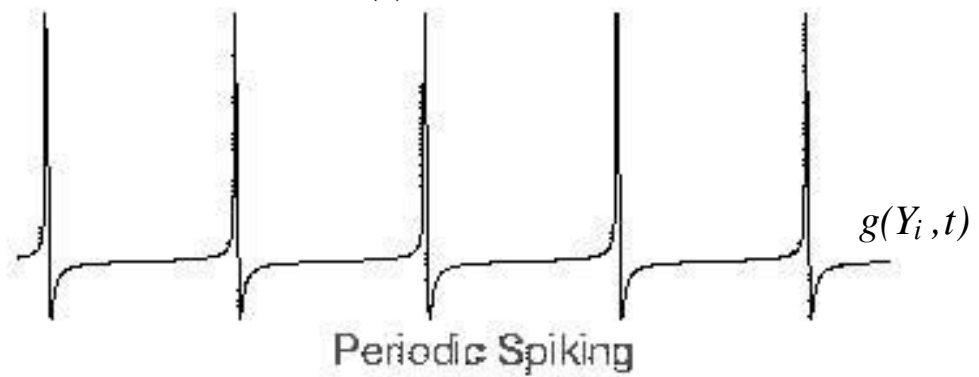

(b)

Fig. 2. The neuronal response of TR neuron. (a) When a periodic bursting pattern (fast burst of spikes at the $A_{\max }$ and $A_{u p}$ with an aftereffect) is produced by the NRT inhibition. (b) When a periodic spiking pattern is produced. (Source: Adapted from Izhikevich, 2000.)

As mentioned before, ?( $t)$ becomes 1 and $G_{k}(t)$ is increased by $F . Z_{i}$ at the point of $A_{\text {min }}$. Lower limit point of $A$ in its oscillation, in which the sign of derivative of $A$ is changed, is calculated by:

$$
A_{l o w}=A_{\min }-\eta \cdot \mid \operatorname{Min}\left[\left(G_{k}^{A_{m n}}+\phi \cdot Z_{i} \cdot\left(1-e^{-t / \varepsilon}\right)\right)-G_{k}(0)\right] \cdot\left(1-e^{-t / \varepsilon}\right)
$$


$A$ is moved from $A_{\text {low }}$ to $A_{\max }$ and then to $A_{u p}$ provided that:

$$
Z_{i}^{c}=\frac{\frac{A_{\max }-A_{\min }}{\eta \cdot\left(1-e^{-t / \varepsilon}\right)}+G_{k}(0)-G_{k}^{A_{m n}}}{\phi \cdot\left(1-e^{-t / \varepsilon}\right)}
$$

$Z_{i}^{c}$ is the same as critical value for the bifurcation of the neuronal response. This system has a limit point attractor for $\boldsymbol{Z}_{\boldsymbol{i}}<\boldsymbol{Z}_{\boldsymbol{i}}^{\boldsymbol{c}}$ and a limit cycle attractor for $Z_{i}>Z_{i}^{c}$

If the firing of TR neuron continues, the habituation effect will be appeared (the sensitivity of the neuron for responding to its input will decreased). This effect could be described by minimal increase in the threshold of the TR neuron. The alternations of the threshold due to habituation effect will be given by:

$$
\alpha \frac{d \theta_{i}^{2}}{d t}=-\theta_{i}^{2}+g\left(Y_{i}, t\right)
$$

Where $a$ is a time constant, which is larger than $e$ so $\boldsymbol{\theta}_{\boldsymbol{i}}^{2}$ will be alternated slowly.

$\boldsymbol{\theta}_{i}^{2}(\boldsymbol{t})$ has also oscillatory behavior, which causes to change the total threshold in Eq.(1). $\boldsymbol{\theta}_{\boldsymbol{i}}^{2}(\boldsymbol{t})$ decreases in the inter-spike interval. A possible mechanism for neuronal habituation is decrease in post-synaptic receptors (down regulation of receptors). The number of post-synaptic receptors after habituation could be computed by the following function:

$$
N=N_{0} \cdot e^{-\alpha \cdot g\left(Y_{i}\right) . t}
$$

Where $N_{O}$ is the initial number of receptors. Down regulation of GABA receptors in LGN (7) decreases the inhibitory effect of NRT on TR neurons. This phenomenon dominantly occurs in the magnocellular stream during transient visual attention (8). The results of computer simulation for our bursting model are shown in Fig. (1).

\section{Discussion}

This neurobiological modeling of bursting response has been described in this article, which its main part is studying the events related to $\mathrm{Ca}^{2+}$-dependant $\mathrm{K}^{+}$ channels. The basic concepts of our bursting model are extracted from Macgregor's model for single neurons (9). 
NRT and thalamus have a basic role in producing bottom-up saliency map during pre-cueing visual attention task. When an abrupt onset cue is presented in the visual field, it makes an intensity change, which is detected by the retinal and thalamic cells. The corresponding spatial location in the NRT becomes salient by the competitive network. The magnification of the neuron is an index of saliency in NRT. The winner neuron in the competition sends a signal to corresponding thalamic neuron, which causes it to produce bursting response (very rhythmic and synchronous action potential discharges are generated during bursting phase (10)). As a result, cued location becomes salient in the thalamus like NRT. This saliency encodes to the cortex after NRT and thalamic computations. On the basis of Niebur et al's model (11), the synchronous thalamocortical oscillations of the neurons whose receptive fields overlap with the focus of attention suppress the response of other cortical neurons associated with non-attended visual stimuli.

Oscillatory behavior of bursting response has a biphasic response time pattern. Each period of this oscillation consists of two parts, which the saturation point of the response function $(A)$ increases in one of them and decreases in another. The first part could be considered as stimulus onset asynchrony (SOA) and the second part as inhibition of return (IOR). After cue presentation, $A$ increases until reaches $A_{u p}$. If target is presented at this time, the most facilitation for transformation of information (the best priming of attended location) is made because the firing rate of thalamic neurons is maximal. As a result, the performance will be enhanced for this SOA. A suitable SOA is needed for producing a good saliency map. Physiological studies have shown that the activation of $\mathrm{Ca}^{2+}$-dependent $\mathrm{K}^{+}$conductance elongates $200 \mathrm{msec}$ (12). It means that the hyperpolarization must be elongated in order to have the bursting response. On the other hand, the best SOA for target detection is 200 msec confirmed by the psychophysical experiments (13). The time constant (e) in the model could be determined so that this SOA is satisfied. This is a neurobiological implication for SOA in our model.

SOA is different for cued targets presented at various distances from the fixation point. The further the target from fixation, the longer the SOA at which the reaction time function reaches asymptote (14). This phenomenon can not be absolutely explained by bottom up saliency map representation in thalamus because there is no difference among bursting patterns of thalamic neurons in response to signals of the cue presented at different spatial locations (15). It seems that another mechanism should exist besides the described mechanism for the SOA. The longer SOA for further cues from the fixation point could be due to the delay needed for the shifting of the attentional spot light. Cue causes corresponding thalamic neurons to produce bursting response by the bottom-up mechanism and leads attentional spot light to move to salient location by the top-down mechanism. The shifting of the spot light is analog $(16,17)$. In other words, the spot light of attention, which is about 1 degree of visual angle in size (18) moves across the visual field in an analog fashion and travels through space at a constant velocity of about 1 degree per $8 \mathrm{msec}$ (14) however there is also evidence that it can vary in size and velocity of movement. The size of attentional focus depends on the attentional load. Perhaps, when attending to a single item, the extra attentional resources would allow a further tightening of the attentional field (i.e., a finer resolution of attention) (19). We suggested the possible neuronal mechanism for the shifting of the spot light in the discussion part, which pulvinar has a critical role in this procedure. 
The amount of the saturation point $(A)$ decreases during the second part of the oscillation period so the thalamic neuron will not produce any response in the interspike interval. This is the inhibitory aftereffect of bursting response, which could be considered as the IOR. The performance of target detection will be poor when the target is presented at the time of IOR. As Jahnsen and Llinás showed before $(2,3)$, the aftereffect of bursting response elongates 80-150 msec.

\section{References}

1) Llinás R., Jahnsen H. (1982). Electrophysiology of mammalian thalamic neurons. Nature (London), 297, 406-8.

2) Jahnsen H., Llinás R. (1984). Electrophysiological properties of guinea-pig thalamic neurons: an in vitro study. J Physiol, 349, 205-26.

3) Jahnsen H., Llinás R. (1984). Ionic basis for the electro-responsiveness and oscillatory properties of guinea-pig thalamic neurons in vitro. J Physiol, 349, 227 47.

4) Taylor J.G., Alavi F.N. (1995). A global competitive neural network. Biol Cybern, 72, 233-48.

5) Crick F. (1984). Function of the thalamic reticular complex: The searchlight hypothesis. Proc Natl Acad Sci, 81, 4586-90.

6) Izhikevich E.M. (2000). Neural excitability, spiking and bursting. Int J Bifur Chaos, 10, 1171-266.

7) Hendry S.H., Miller K.L. (1996). Selective expression and rapid regulation of GABAA receptor subunits in geniculocortical neurons of macaque dorsal lateral geniculate nucleus. Vis Neurosci, 13, 223-35.

8) Steinman B.A., Steinman S.B., Lehmkuhle S. (1997). Transient visual attention is dominated by the magnocellular stream. Vis Res, 37, 17-23.

9) MacGregor R.J. (1987). Neural and brain modeling. Academic Press, San Diego, California.

10) Bal T., McCormick D.A. (1993). Mechanisms of oscillatory activity in guinea-pig nucleus reticularis thalami in vitro: a mammalian pacemaker. J Physiol (London), 468, 669-91.

11) Niebur E., Koch C.,Rosin C. (1993). An oscillation-based model for the neuronal basis of attention. Vis Res, 33, 2789-802.

12) Sherman S.M., Koch C. (1986). The control of retinogeniculate transmission in the mammalian lateral geniculate nucleus. Exp Brain Res, 63, 1-20.

13) Nakayama K., Mackeben M. (1989). Sustained and transient components of focal visual attention. Vis Res, 29, 1631-47.

14) Tsal Y. (1983). Movements of attention across the visual field. J Exp Psychol Hum Percept Perform, 9, 523-30.

15) Hammond C. (1996). Cellular and molecular neurobiology. Academic Press, San Diego, California.

16) Chastain G. (1992). Time-course of sensitivity changes as attention shifts to an unpredictable location. J Gen Psychol, 119, 105-11.

17) Shepherd M., Muller HJ. (1989). Movement versus focusing of visual attention. Percept Psychophys, 46, 146-54.

18) Eriksen B.A., Eriksen C.W. (1974). Effects of noise letters upon the identification of a target letter in a nonsearch task. Percept Psychophys, 16, 143-9.

19) Intriligator J., Cavanagh P. The spatial resolution of visual attention. Cog Psychol, In Press. 